\title{
7 Zusammenfassung der wichtigsten Informationen aus Teil I „Grundlagen“
}

In Teil I „Grundlagen“ wurde eine möglichst praxisnahe Erläuterung des Qualitätskonstrukts in der ambulanten medizinischen und psychotherapeutischen Patientenversorgung vorgenommen, sowie aktuelle Vorgaben zum systematischen Qualitätsmanagement vorgestellt:

- Gute Qualität in der ambulanten Patientenversorgung ist ein komplexes Konstrukt, das auf der Grundlage von Anbieter-, Nutzern und Kostenträgeranforderungen entsteht und bewertet wird.

- Jede Praxis muss selbst definieren, wie sie ihre Versorgungsqualität definiert.

- Es besteht für alle ärztlichen, psychotherapeutischen und kinderpsychotherapeutischen Praxen und MVZ, die im Rahmen der GKV Leistungen erbringen und abrechnen, die Verpflichtung zum praxisinternen Qualitätsmanagement, dargelegt in der QM-Richtlinie des Cemeinsamen Bundesausschusses.

- Eine Zertifizierung ist nicht vorgeschrieben.

- Organisationen, die ihre Verbesserungsprozesse kontinuierlich reflektieren und steuern, also Qualitätsmanagement systematisch umsetzen, entwickeln ein hohes Maß an Zufriedenheit der Mitarbeiter und Patienten, Sicherheit und Wettbewerbsfähigkeit. Diese Vorteile gehen weit über das hinaus, was eine Organisation erzielt, wenn die formalrechtlichen Vorgaben mehr oder weniger widerwillig „abgearbeitet“ werden. 